\title{
COMPARANDO CON LAS GRANDES. RETOS PARA LAS ASEGURADORAS EN REDUCCIÓN DE COSTOS
}

\author{
Hugo Javier Fuentes Castro* \\ Ana María Reyna Bernal ${ }^{* *}$
}

(Recibido: marzo 2014/Aceptado: junio 2014)

\section{Resumen}

Este artículo utiliza la descomposición de las fuentes económicas de las variaciones de los costos para examinar las características de las empresas eficientes de la industria de seguros en México. A fin de asegurar consistencia en la comparación, el mercado se segmentó en tres grupos de acuerdo a su especialidad, y se presenta en el ejercicio de comparación, una o más empresas eficientes en cada grupo. Siguiendo un modelo basado en el análisis de frontera no paramétrico, la diferencia en el costo de las empresas eficientes respecto a sus pares se descompone en el efecto de los precios, la escala, la eficiencia técnica y la eficiencia asignativa. De esta forma se hacen evidentes las ventajas de las aseguradoras más eficientes en costos. El análisis, de corte transversal, fue aplicado a datos del periodo 2008-2012 para observar la evolución de las empresas eficientes respecto a su grupo durante cinco años.

Palabras clave: seguros, eficiencia en costo, DEA.

Clasificación JEL: C14, C67, D61, G14, G22.

\footnotetext{
* Profesor-investigador del Instituto Tecnológico y de Estudios Superiores de Monterrey, Campus Ciudad de México Dirección para correspondencia: Calle del Puente núm. 222, col. Ejidos de Huipulco, Tlalpan, CP 14380, México, DF. <hfuentes@itesm.mx>, oficina 54832223.

* Profesora del Instituto Tecnológico y de Estudios Superiores de Monterrey, Campus Ciudad de México Dirección para correspondencia: Cerro de las Torres, 393, Campestre Churubusco, CP 04200, Coyoacán, México, DF, <ana.reyna@gnp.com.mx>, <A0332085@itesm.mx>, oficina 5227 3354, móvil 5532252121.
} 


\section{Abstract}

This paper utilizes the decomposition of economic sources of cost variations to examine efficient firms' characteristics in the insurance industry in Mexico. In order to assure consistent comparison, this market was segmented into three groups according to their specialization, and one or more efficient companies of each group are presented in the benchmarking exercise. Following a model based on a non-parametric frontier analysis, the difference in cost, between efficient firms and their peers, is decomposed into: input price, scale, technical efficiency and allocative efficiency effects. In this way, advantages of more cost efficient insurers become evident. The cross-sectional analysis was applied to 2008-2012 data to observe evolution of efficient companies regarding their group during five years.

Keywords: insurance, cost efficiency, DEA.

JEL Classification: C14, C67, D61, G14, G22.

\section{Introducción}

En el contexto, ya complejo para el sector asegurador, de reducción de la actividad económica y su recuperación del 2008 al 2012, las aseguradoras en México han tenido que enfrentar, en adición, otros retos y oportunidades a la vez. El mercado asegurador mexicano ha mantenido un crecimiento superior al de la economía del país, lo que contribuye a la atracción de nuevos competidores, intensificando las presiones en los precios y, por tanto, menores márgenes. Por otra parte, la conducta del consumidor se está moviendo a canales menos tradicionales y con mayor prioridad al precio que al servicio, demandando a las aseguradoras productos más simples y accesibles en costo. ${ }^{1}$

En palabras de Borenstein y Farrell (2000), en respuesta a las presiones financieras, los administradores reducen la ineficiencia, con la optimización de los procesos o cortando costos o "grasa", ${ }^{2}$ ya sea a partir de cambios en

\footnotetext{
${ }^{1}$ Accenture (2014) relata tendencias globales en los cambios de los asegurados, en especial en las clases medias, el potencial de expansión que esto representa para las aseguradoras, y las implicaciones en su operación: eficiencia en costo y desarrollo de economías de escala.

${ }^{2}$ Borenstein y Farrell (2000, p. 224).
} 
sus prácticas de gestión, el control de los desperdicios, la introducción de tecnologías ahorradoras en costo, o inclusive reduciendo la calidad del servicio. Con o sin inversiones de capital adicionales, las empresas se acercan a las mejores prácticas con estas acciones.

En el campo de la economía, la frontera de costos teórica captura el estándar de las mejores prácticas de la industria y, junto con el concepto de eficiencia en costo, es posible estimar en cada empresa el desperdicio en insumos y la asignación inadecuada de los mismos dados sus precios, respecto a las posibilidades de producción del mercado.

La eficiencia en costo de la industria de seguros es un campo poco estudiado en comparación a la eficiencia técnica, donde podemos encontrar múltiples métodos aplicados en una amplia gama objetivos de investigación y de mercados analizados. La investigación que aquí se presenta, contribuye al análisis del desempeño económico del sector asegurador en México, a través de un análisis de frontera con datos transversales, con el objetivo de distinguir las ventajas de las empresas eficientes a partir de la descomposición de sus costos y las brechas con sus pares. Siguiendo a Grifell-Tatjé y Lovell (2000), el método para comparar el desempeño de empresas usando la frontera de costos es un análisis poco usual que apuntará a diferencias en variables exógenas, como es la diferencia en tamaño, y también a variables endógenas, bajo el control de los administradores.

Entre las investigaciones en el sector asegurador que preceden a esta investigación existen trabajos que estiman la eficiencia en costo y su relación con otras variables. Como ejemplo, en el campo de los métodos paramétricos, Cummins y Weiss (1993) estudian las empresas de seguros dedicadas a la cobertura de propiedades en el mercado norteamericano durante la década de los ochentas, con el objetivo de determinar si la inflación en el precio de las pólizas de seguros se relaciona con la ineficiencia en costo. Rai (1996) analiza la eficiencia en costo de aseguradoras multinacionales con presencia en 11 países; y Jameti y Von Ungern-Sternberg (2005) comparan la eficiencia en costo de empresas públicas y privadas de Suiza. Por otra parte, con métodos noparamétricos encontramos, por ejemplo, la comparación de la eficiencia en costo de compañías aseguradoras alemanas de acuerdo a sus canales de distribución en Trigo-Gamarra y Growitsch (2010), y la evaluación de la relación entre las condiciones de gobierno corporativo y la eficiencia en costo de las empresas Takaful en países islámicos en Kader, Adams y Hardwick (2010). Turchetti y Daraio (2004), también en el campo de los métodos 
no paramétricos, estiman la evolución de la eficiencia en costo, el cambio tecnológico, la eficiencia técnica y la eficiencia en escala de las aseguradoras de automóviles en Italia.

En México, Fuentes (1993), Ugalde (1993) y Huidobro (2002) valoran con métodos paramétricos la eficiencia en costo de las aseguradoras para explorar la existencia de economías de escala y alcance, en las décadas de los ochentas y noventas, antes de la apertura total del mercado a empresas con capital cien por ciento extranjero.

A diferencia de los estudios mencionados, esta propuesta busca aproximarse a las fuentes económicas de las diferencias en el costo de los insumos siguiendo la formulación de Grifell-Tatjé y Lovell (2000). El diferencial de costo entre las empresas eficientes y el promedio de sus pares se descompone en dos etapas en este modelo. La primera, busca la contribución aislada del efecto de los precios y de las cantidades al costo, y la segunda, descompone el efecto de las cantidades en las contribuciones de la escala o tamaño de la operación, la eficiencia técnica y la eficiencia asignativa.

La cantidad de insumos utilizada es capturada por la eficiencia técnica, y la combinación óptima de insumos de acuerdo a sus precios para minimizar el costo, se estima en la eficiencia asignativa. En una primera instancia, la descomposición aporta información para explicar la ventajas de las empresas eficientes en costo, en segundo término permite llegar a conclusiones del desempeño del sector.

El modelo de Grifell-Tatjé y Lovell (2000), a saber de los autores del presente documento, no ha sido aplicado en la industria de seguros. En el artículo seminal se evaluaron compañías productoras de energía eléctrica, y posteriormente fue aplicado el modelo en el análisis del desempeño de los trenes Clase I de los Estados Unidos en Lim y Lovell (2008).

La investigación, aunque aplica metodologías orientadas a los insumos, parte de la concepción que las aseguradoras operan bajo una tecnología de producción conjunta de productos de protección financiera y de servicios de compensación de pérdidas financieras originadas por el riesgo puro, en el entendido que las aseguradoras tienen motivaciones para expandir su participación en el mercado y a la vez controlar la frecuencia y severidad de la siniestralidad (Schlesinger y Venezian, 1986; Ben-Shahar y Logue, 2012). Por esta razón, los siniestros reciben el tratamiento de producto indeseado, y las pólizas de seguros el de productos deseados. El enfoque metodológico de esta investigación también es una aportación a la literatura del análisis 
de la industria de seguros, al ser distinto a la mayoría de las investigaciones del sector. En los modelos donde la siniestralidad es el producto de la industria de seguros, las empresas con problemas de solvencia por mala gestión de sus riesgos podrían obtener los indicadores más altos de productividad y eficiencia, situación sin sentido económico (Triplett y Bosworth, 2004), además, sus resultados son afectados por la volatilidad y variabilidad de este producto negativo.

Las empresas incluidas en la investigación son todas las aseguradoras con actividad en México entre 2000 y 2012, excepto aquellas clasificadas por la Comisión Nacional de Seguros y Fianzas en una categoría diferente a seguros, esto es, las dedicadas a pensiones, salud, garantías financieras y reaseguradoras.

Para fines del análisis se formaron tres grupos de empresas usando la clasificación del Banco de México: ${ }^{3}$ i) vida, cuando emiten pólizas para las personas, esto es de los ramos de vida y gastos médicos y accidentes; ii) no vida, si emiten pólizas para los bienes que corresponde a las pólizas de automóviles y al resto de los ramos de daños y, iii) mixtas, cuando emiten pólizas tanto de vida como de no vida. La agrupación de las aseguradoras acorde a su especialización aumentará la similitud de la tecnología de producción de las instituciones de cada grupo, con la ventaja de crear una frontera empírica más ajustada a partir de las observaciones por la técnica no paramétrica análisis envolvente de datos denominada DEA por sus siglas en inglés (Data Envelopment Analysis).

Los resultados proporcionados por esta investigación pueden ser de valor para los accionistas y administradores de las instituciones, porque les dan guías de cuáles acciones podrían incluir en su gestión para mejorar su desempeño y, en consecuencia, su competitividad. Para los reguladores el estudio genera visibilidad de las brechas en el desempeño del sector $\mathrm{y}$, por tanto, del espacio de mejora que podría trasladarse a la sociedad.

El documento se organiza de la siguiente forma: la Sección II describe el modelo de la tecnología de producción y la función de costo para la industria de seguros; la Sección III narra la metodología de estimación para el análisis transversal entre las empresas eficientes y su grupo; la Sección IV está dedicada a la definición de los insumos y productos requeridos para la

\footnotetext{
${ }^{3}$ Banco de México, 2007, p. 162.
} 
estimación de los determinantes de la variación en el costo de los insumos y las fuente de donde se obtuvieron los datos de las variables; la Sección V presenta los resultados más significativos de los análisis para 2012; la Sección VI describe la tendencias observadas al aplicar el análisis transversal de 2008 a 2012, por último, se ofrecen conclusiones en la Sección VII.

\section{Marco conceptual de la tecnología de producción y la función de costos}

Tomando de la eficiencia medio ambiental la tecnología de producción con externalidades, el modelado de la industria aseguradora incluye dos productos, el primero es la cobertura de riesgo comercializada en la forma de pólizas de seguros para representar el producto deseado (good output), y el segundo es el servicio del pago de pérdidas financieras, también llamado pago de siniestro, considerado producto no deseado (bad output) en esta investigación.

Para representar ahora, la tecnología de producción expresada en términos del conjunto de insumos, asuma el lector un vector de insumos no negativos $x=\left(x_{1}, \ldots, x_{N}\right) \in R_{+}^{N}$ y un vector de productos que $u=\left(u_{1}, \ldots, u_{N}\right) \in R_{+}^{M}$ representa la unión del subvector no negativo de productos deseados $y=\left(y_{1}, \ldots, y_{N}\right) \in R_{+}^{M}$ y del subvector no negativo de productos no deseados $b=\left(b y_{1}, \ldots, b_{N}\right) \in R_{+}^{M}$. Siguiendo a Grifell-Tatjé y Lovell (2000), la representación de la tecnología se expresa en la forma (2.1), incluyendo todos los productos deseados e indeseados que pueden producirse con un vector dado de insumos.

$$
L^{t}\left(u^{t}\right)=\left\{x^{t}: x^{t} \text { puede producir } u^{t}\right\}
$$

Se asume que el conjunto de insumos es cerrado, convexo y con disponibilidad fuerte de los insumos $\left[x^{t} \in L^{t}\left(u^{t}\right) \Rightarrow x^{\prime} \in L^{t}\left(u^{t}\right), x^{\prime} \geq x^{t}\right]$.

Todos los vectores $x^{t}$ pertenecerán a su conjunto contemporáneo $L\left(u^{t}\right)$, aunque no todos estarán en la isocuanta de insumos definidas por:

$$
I^{t}\left(u^{t}\right)=\left\{x^{t}: x^{t} \in L\left(u^{t}\right), \lambda x^{t} \notin L^{t}\left(u^{t}\right), \quad \lambda<1\right\}
$$

Para todos los vectores de insumos $x^{t}$ es posible estimar su distancia a la isocuanta de insumos con la función de distancia radial expresada en (2.3).

$$
D^{t}\left(x^{t}, u^{t}\right)=\max \left\{\theta: x^{t} / \theta \in L^{t}\left(u^{t}\right)\right\}
$$


Si el vector $x^{t}$ pertenece a la isocuanta, la función de distancia radial $D^{t}\left(x^{t}, u^{t}\right)$ será igual a uno, y tendrá el valor mayor a uno para el resto de vectores de insumos que pertenecen a $L^{t}\left(u^{t}\right)$.

El modelo utiliza el dual de la función de distancia de insumos, esto es, la frontera de costos. Mientras que la frontera de producción conjunta señala la mejor forma técnica de producción, la frontera de costos describe la forma más económica de ejecución, con un estándar para medir el desempeño económico de las compañías (Kumbhakar y Lovell, 2003).

$$
\begin{aligned}
& c^{t}\left(w^{t}, u^{t}\right)=\min _{x}\left\{\left(w^{t T} x\right): x \in L^{t}\left(u^{t}\right)\right\} \\
& =\min _{x}\left\{\left(w^{t T} x\right): D^{t}\left(x, u^{t}\right) \geq 1\right\}, t=1, \ldots, T
\end{aligned}
$$

Dicha frontera indica el costo mínimo requerido para producir los vectores de productos $u^{t}$, con los vectores de precios estrictamente positivos $w^{t} \mathrm{y}$ las cantidades de insumos $x^{t}$ bajo la tecnología vigente. Los costos de cada compañía estarán en o por debajo de $c^{t}\left(w^{t}, u^{t}\right)$.

La frontera de costos cumple las siguientes propiedades:

$$
\begin{aligned}
& c(0, w)=\{0\} \text { y } c(u, w)>0 \text { para } u \geq 0 \\
& c(u, \lambda w)=\lambda c(u, w) \text { para } \lambda \geq 0 \\
& c\left(u, w^{\prime}\right) \geq c(u, w) \text { para } w^{\prime} \geq w \\
& c(u, w) \text { es una función cóncava en } w \\
& c(u, w) \text { es una función continua en } w \\
& c\left(u^{\prime}, w\right) \leq c(u, w) \text { para } 0 \leq u^{\prime} \leq u
\end{aligned}
$$

De aquí que esta función asume la existencia de costos para la generación de productos (2.5); es homogénea en grado uno, aumenta proporcionalmente al incremento en los precios de los insumos (2.6); no es decreciente para los precios $w$ (2.7); incluye a todas las combinaciones lineales de precios y cantidades por debajo de la curva y por tanto son factibles las combinaciones de insumos menores a las que forman la frontera (2.8); garantiza la existencia 
de un punto que minimiza la función al ser continua (2.9), y permite libre disponibilidad de los productos (3.9). Al cumplir las condiciones (2.52.10) la función de costos es dual de la función de producción, de esta manera es posible inferir aspectos de la estructura de la tecnología de producción a partir de la función de costos, asumiendo también el objetivo de minimizar los costos ante precios determinados exógenamente.

Las compañías ubicadas en el costo mínimo en la frontera se caracterizan por ser eficientes, tanto técnicamente por consumir la cantidad mínima de insumos, como asignativamente por la combinación apropiada de insumos y precios que minimiza el costo.

\section{Marco metodológico de análisis respecto a la práctica eficiente}

Grifell-Tatjé y Lovell proponen utilizar un método no paramétrico para llevar a cabo la comparación ex post del desempeño de las empresas respecto a la práctica eficiente. Con este análisis es calculada la brecha entre las instituciones de seguros y su desempeño potencial si son eficientes, y tienen los precios y la escala de la empresa eficiente u objetivo de su grupo. El comparativo aporta a los administradores información valiosa para su gestión, tanto de la empresa seleccionada como mejor práctica para entender y mantener sus fortalezas, así como del resto de las firmas, ya que algunos de los determinantes de las variaciones en el costo pueden gestionarse a través de las estrategias de competitividad de cada compañía. Por otra parte, se proporciona información del desempeño de la industria, de posible interés para los reguladores, con datos cuantificados sobre las brechas en desempeño con relación a las posibilidades de producción de la tecnología vigente, dejando en evidencia la oportunidad de mejora en el mercado que podría traducirse en beneficios para los consumidores.

El modelo es construido en dos etapas, en la primera descomposición, expresada en (3.1), la diferencia entre el costo de los insumos de la empresa eficiente y el promedio del grupo es dividida en la contribución de los precios ponderados por las cantidades, y en la aportación de las cantidades de insumos ponderada por los precios.

Para representar el modelo se considera en un periodo de tiempo el número de empresas $I$, donde una de ellas, seleccionada por ser eficiente, establecerá la meta de costo $c^{*}$ y el resto participará con su costo $c^{o}$ en 
(3.1). Note la ponderación de la diferencia en precios y cantidades por los indicadores del tipo Bennet $\bar{x}=\frac{1}{2}\left(x^{o}+x^{*}\right) \mathrm{y}_{\bar{w}}=\frac{1}{2}\left(w^{o}+w^{*}\right) \cdot{ }^{4}$

$$
\begin{array}{lr}
c^{o}-c^{*}=w^{o T} x^{o}-w^{* T} x^{*} & \\
\bar{x}\left(w^{o}-w^{*}\right) & \text { efecto precio } \\
\bar{w}\left(x^{A}-x^{*}\right) & \text { efecto escala }
\end{array}
$$

El primer componente del modelo, el efecto precio, indica el diferencial entre el costo promedio de las empresas del grupo y el costo de la empresa eficiente, explicado por las diferencias en los precios de los insumos. Tendrá signo negativo si la empresa eficiente paga precios más altos y viceversa, si en promedio, el resto de las empresas paga precios menores. El segundo componente de este análisis es el efecto de la escala, ilustrado en la figura 1 , con el dimensionamiento de la brecha entre el costo con las cantidades de insumos de la empresa eficiente $x^{*}$ y el costo promedio de las cantidades óptimas para minimizar el costo del grupo $x^{A}$. El signo es positivo del efecto escala si la empresa eficiente consume menor cantidad de insumos que el óptimo del grupo necesario para minimizar costos, y es negativo si la empresa eficiente tiene un costo mayor que el resto de las empresas de su grupo originado por la cantidad de insumos que emplea.

El tercer y cuarto componente del análisis son la eficiencia técnica y la eficiencia asignativa en (3.2). El efecto eficiencia técnica nos indica la diferencia promedio en costo entre las cantidades observadas de las empresas de cada grupo y la proyección en la frontera eficiente. Resultará positivo este efecto si las empresas del grupo son en promedio ineficientes técnicamente, en tanto, su costo aumentará por usar mayor cantidad de insumos de lo marcado por la tecnología. El efecto eficiencia técnica, podría tener valor cero si todas empresas del grupo fueran eficientes técnicamente, este efecto no genera valores negativos bajo este modelo transversal.

$$
\begin{array}{ll}
\bar{w}\left(x^{o}-x^{C}\right) & \text { efecto eficiencia técnica } \\
\bar{w}\left(x^{C}-x^{A}\right) & \text { ef ecto eficiencia asignativa }
\end{array}
$$

\footnotetext{
${ }^{4}$ Son indicadores por calcularse con diferencias en tanto, los índices se expresan con divisiones, en la literatura la descomposición en el efecto de los precios frecuentemente utiliza indicadores o índices Laspeyres y Paasche. Los indicadores Bennet sin preferibles para evitar ponderaciones en exceso con los periodos con niveles más altos de precios o cantidades, en especial en las comparaciones con diferencias importantes. La demostración axiomática de esta ventaja de los indicadores Bennet se encuentran en Diewert (2005) y la representación gráfica en Sahoo y Tone (2009).
} 
El efecto eficiencia asignativa mide la inadecuada asignación de insumos de acuerdo a sus precios. Su magnitud con signo positivo indica qué tan lejos están las empresas del grupo, en términos de costo, respecto a las cantidades que lo minimizan. Asimismo, el efecto eficiencia asignativa podría ser cero si todas las empresas del grupo operaran con las cantidades óptimas de insumos para minimizar sus $\operatorname{costos}\left(x^{o}=x^{A}=x^{C}\right)$.

\section{Figura 1}

Comparativo transversal con empresa eficiente

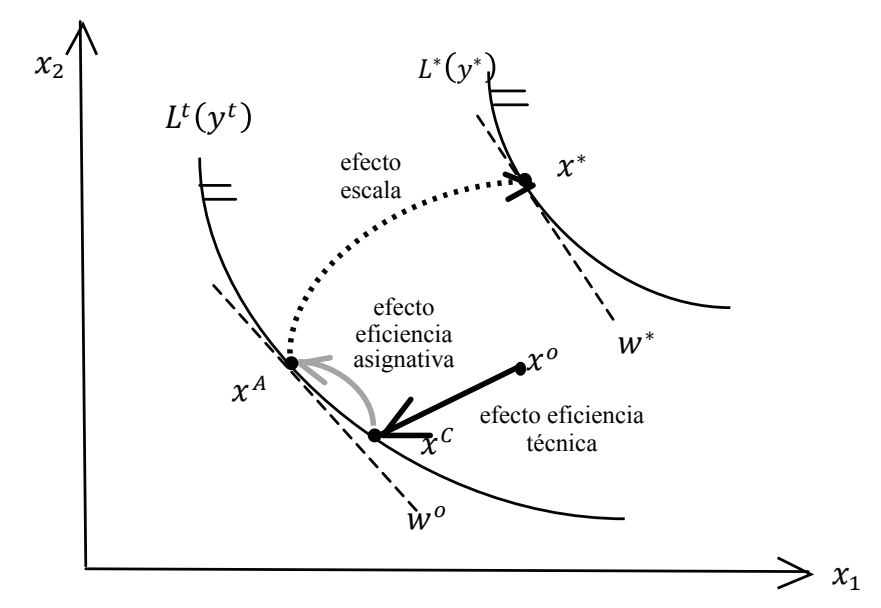

Fuente: elaboración propia.

El modelo se resuelve al encontrar los dos vectores no observados con programas lineales utilizando los datos de precios y cantidades de insumos la empresa y las cantidades de sus productos. El vector $x^{A}$ es estimado con el programa lineal (3.3) cuyo objetivo es encontrar las cantidades de insumos que minimizan los costos. Las restricciones del programa satisfacen $w^{o T} x^{A}=c\left(u^{o}, w^{o}\right)$, además de permitir rendimientos variables a escala.

$$
\min \left\{w^{t T} x: x^{t} \geq X^{t} \lambda, U^{t} \lambda \geq u^{t}, \lambda \geq 0, \Sigma \lambda=1\right\}
$$

Ahora bien, el vector $x^{C}$ es la proyección de $x^{o}$ sobre la frontera eficiente obtenida a partir de la contracción radial de los insumos como se muestra 
en la figura 1. El cálculo de este vector se resuelve con el programa lineal (3.4) al insertar el valor de $\theta$ estimado en $x^{C}=\theta^{C} x^{t}=x^{t} / D^{t}\left(x^{t}, u^{t}\right)$.

$$
\min _{\theta}\left\{\theta: \theta x^{t} \geq X^{t} \lambda, U^{t} \lambda \geq u^{t}, \lambda \geq 0, \Sigma \lambda=1\right\}
$$

Es importante destacar que en los programas (3.3) y (3.4) los productos deseados e indeseados son tratados en forma asimétrica siguiendo la propuesta de Seiford y Zhu (2002) y Zhu (2009), al aprovechar la propiedad del DEA de clasificación de eficiencias e ineficiencias sin importar la transformación de los datos. Los productos indeseados son procesados en los programas lineales de igual forma que los productos deseados después de una transformación monotónica lineal al multiplicarlos por "-1" y al agregar un valor v suficiente para convertir los valores negativos en positivos. Siendo y el subvector de los productos buenos, y b el de los productos malos, la transformación se logra con $\bar{b}=-b+v>0$, donde $v=\max \{b\}+1$.

\section{Datos}

Los modelos descritos se resuelven con precios y cantidades de insumos y productos construidos a partir de los estados financieros y los reportes anuales de la Comisión Nacional de Seguros y Fianzas (CNSF), órgano regulador del sector asegurador en México. Para aquellas empresas sin datos disponibles en los reportes anuales de la CNSF se obtuvo la información de bases de datos proporcionados por la Asociación Mexicana de Instituciones de Seguros (AMIS).

Para caracterizar las cantidades de los insumos existe en la literatura poca divergencia, en la mayoría de los estudios analizados el trabajo, el capital y otros gastos de operación son considerados insumos de la industria aseguradora. En el insumo trabajo, algunos autores agregan datos de los agentes, sean o no empleados de las compañías. ${ }^{5}$ En la definición del capital se han utilizado representaciones tanto del capital físico, como del capital financiero, y en cuanto a otros gastos de operación, algunos autores consideran los gastos de administración sin los costos del

\footnotetext{
${ }^{5}$ Ejemplos en Bernstein (1999), Fuentes, et al. (2001), Barros, et al, (2008), Garg y Deepti (2009) y Mahlberg y Url (2010).
} 
trabajo y otros agrupan ambos costos, cabe señalar que la tercerización de servicios se recoge en este insumo.

Desde el enfoque financiero, Cummings y Mahul (2009) descomponen la prima de seguros en costos del capital, gastos y siniestralidad esperada. Este enfoque, si bien está orientado a señalar los costos que incurre una aseguradora, incluyendo el pago al accionista, nos ayuda a hacer una transición entre el costo de los insumos y los gastos del sector. En la industria aseguradora, los gastos se dividen en gastos de administración, gastos de adquisición y gastos de ajuste. ${ }^{6}$ En los de adquisición, están los costos de los intermediarios, tanto bonos y comisiones como el resto de los costos asociados a la venta. Los gastos de ajuste comprenden las estructuras, instalaciones, servicios contratados y otros insumos destinados a la atención de siniestros sin incluir el pago de indemnizaciones. Finalmente, en gastos de administración se registra el número de empleados fijo de la compañía y el resto de los insumos para la operación no asociados a la venta ni al ajuste.

Atendiendo el punto de vista económico de los insumos y el financiero de gastos de la compañía, esta investigación aplicará cuatro insumos: empleado $w_{1} x_{1}$ e intermediario $w_{2} x_{2^{\prime}}$ para representar el trabajo como recurso necesario para la operación de las aseguradoras; y el resto de los insumos dividido en otros gastos de administración $w_{3} x_{3}$ y en gastos de ajuste $w_{4} x_{4}$. Esta propuesta, además de aproximarse con datos de los estados de resultados en forma directa, ayuda a examinar el papel de cada tipo de gasto en la presentación de resultados.

Sin duda la cantidad del insumo empleado puede representarse con el número de empleados, información disponible a través de la AMIS; sin embargo, la información fue descartada porque el 18\% de las observaciones mostraba empresas con cero, uno o dos empleados. Por otra parte, una revisión de las cifras de gastos de operación reportadas en los estados de resultados conduce a concluir que algunas empresas transfirieron a sus empleados a subsidiarias y aumentaron la contratación de fuerza de trabajo a terceros. Por lo anterior, el costo total de este insumo se aproxima en esta investigación con el monto de remuneraciones y

\footnotetext{
${ }^{6}$ La regulación en México también marca el registro, otro tipo de gastos no relacionados directamente con la operación de la prima de riesgo, por ejemplo los gastos por servicios análogos y conexos asociados a servicios complementarios proporcionados por las aseguradoras a sus asegurados, por ejemplo en la administración del copago de servicios médicos. Véase CNSF (2011c.)
} 
prestaciones en adición al monto de honorarios localizados en gastos de operación. El precio $w_{1}$ para este insumo es el aumento anual al salario mínimo, y la cantidad $x_{1}$ es el resultado de la división del costo del insumo entre su precio. Cabe apuntar, que no todo el costo de los empleados de las aseguradoras está registrado en los gastos de administración, luego que en promedio, entre 2 y $4 \%$ de los gastos de adquisición y de ajuste corresponden al costo de empleados dedicados a la venta y a la atención de reclamaciones.

Por otra parte, el costo total del insumo intermediario se aproxima a partir de los renglones de estado de resultados denominados comisiones a agentes, compensaciones adicionales a agentes y otros gastos de adquisición, consiguiendo el monto del gasto de adquisición, neto de los efectos de reaseguro. El precio de los intermediarios $w_{2}$ se calcula con el porcentaje del gasto de adquisición obtenido respecto a los ingresos por venta de pólizas de seguros, que a su vez juega en el modelo como la cantidad del insumo intermediario $x_{2}$.

El insumo otros gastos de administración se estima con la suma de los gastos de operación, las amortizaciones y depreciaciones deduciendo el costo del insumo empleado. El precio $w_{3}$ utilizado para este insumo es el porcentaje de su monto entre el valor de los activos fijos que representarán la cantidad $x_{3}$. En lo que respecta a los gastos de ajuste, su precio $w_{4}$ es calculado con el porcentaje de estos gastos en relación a la siniestralidad directa $x_{4}$.

La cantidad de producto deseado se aproxima entonces con el número de pólizas emitidas y su precio es obtenido con la prima promedio de cada uno de los cuatro ramos: vida, gastos médicos mayores y accidentes, automóviles y daños. En cuanto a la aproximación de los productos indeseados, en forma análoga, el número de siniestros reportados cada año representa la cantidad de este tipo de productos por ramo, y el precio es el costo medio del siniestro obtenido con la división de la siniestralidad por ramo entre el número de siniestros reportados. La tabla 1 resume los insumos y productos utilizados para resolver los modelos de estimación de este estudio. 
Tabla 1

Insumos y productos

\begin{tabular}{|c|c|c|}
\hline \multicolumn{3}{|l|}{ Insumos } \\
\hline \multicolumn{3}{|l|}{ Gastos de administración } \\
\hline \multirow[t]{2}{*}{ Empleado } & $w_{1}$ & variación promedio del salario mínimo ${ }^{7}$ \\
\hline & $x_{1}$ & (\$) costo anual del trabajo divido entre $w_{1}$ \\
\hline \multirow[t]{2}{*}{$\begin{array}{l}\text { Otros gastos de adminis- } \\
\text { tración }\end{array}$} & $w_{3}$ & $\begin{array}{l}\text { (\%) proporción de otros gastos de operación } \\
\text { respecto al valor de los activos fijos }\end{array}$ \\
\hline & $x_{3}$ & (\$) valor de los activos fijos al cierre del año \\
\hline \multirow[t]{2}{*}{$\begin{array}{l}\text { Gastos de adquisición Inter- } \\
\text { mediario }\end{array}$} & $w_{3}$ & $\begin{array}{l}\text { (\%) proporción de los gastos de adquisición } \\
\text { anuales respecto a las primas directas del año }\end{array}$ \\
\hline & $x_{3}$ & (\$) primas directas anuales \\
\hline \multirow[t]{2}{*}{ Gastos de ajuste } & $w_{4}$ & $\begin{array}{l}\text { (\%) proporción del monto anual de gastos de } \\
\text { ajuste respecto al pago de reclamaciones o } \\
\text { siniestralidad directa del año }\end{array}$ \\
\hline & $x_{4}$ & (\$) valor de la siniestralidad directa \\
\hline \multicolumn{3}{|l|}{ Productos } \\
\hline $\begin{array}{l}\text { Pólizas de seguros emitidas } \\
\text { y pagadas (productos desea- } \\
\text { dos) }\end{array}$ & $p_{y}$ & $\begin{array}{l}\text { (\$) prima promedio por línea de productos } \\
\text { (vida, gastos médicos, automóviles y daños) } \\
\text { total de pólizas emitidas y pagadas en el año } \\
\text { por línea de productos }\end{array}$ \\
\hline Siniestros reportados & $p_{b}$ & $\begin{array}{l}\text { (\$) costo medio del siniestros por línea de } \\
\text { productos }\end{array}$ \\
\hline (productos no deseados) & $b$ & $\begin{array}{l}\text { total de siniestros reportados por línea de } \\
\text { productos }\end{array}$ \\
\hline
\end{tabular}

Fuente: elaboración propia.

Fuente: Comisión Nacional de Salarios Mínimos. Tabla de salarios mínimos general promedio de los Estados Unidos Mexicanos. http://www.conasami.gob.mx/pdf/salario_minimo/sal_min_gral_prom. pdf. Última visita 6 de julio de 2014. 


\section{Resultados del análisis respecto a la práctica eficiente 2012}

La aplicación del modelo para comparar la descomposición del costo de cada grupo respecto a una empresa eficiente destaca los aspectos distintivos de la empresa eficiente en relación a cada grupo como conjunto.

El estudio implica definir un criterio para establecer las empresas objetivo. En este caso, fueron seleccionadas la empresas consistentemente eficientes del 2008 al 2012 a partir de los resultados de los programas (3.3) y (3.4). Como se verá en los grupos de empresas de vida y no vida, solamente una empresa cumplió el requisito, mientras que en el grupo de las empresas mixtas, más de una compañía se mantuvo eficiente durante los cinco años.

Metlife fue seleccionada para conducir el comparativo de las empresas de vida en el 2012, sus primas en el 2012 constituyen el 81\% de la emisión de su grupo, y sus activos fijos representan el 79\% de las empresas de vida. La gran diferencia en el tamaño en comparación con sus pares, se refleja en varios componentes de la descomposición del costo de Metlife.

En la tabla 2 se muestra la brecha promedio entre el costo del grupo y Metlife por -5 823 millones de pesos (mdp), este monto y la desviación estándar de 206 mdp revela que el grupo se forma por empresas pequeñas con un costo total promedio mucho menor a Metlife. La brecha por escala en menos 13.218 mdp remarca nuevamente la gran diferencia en tamaño entre Metlife y el resto del grupo, lo cual es el principal componente que explica la diferencia de costo.

Las ventajas del desempeño de la empresa eficiente son reveladas por el análisis, en primer lugar el efecto precio al ser positivo indica que el resto de las compañías pagan en promedio precios de sus insumos superiores a los de Metlife, y las dos últimas columnas de la tabla 2, indican que en una pequeña cantidad, la brecha del costo se explica por la ineficiencia del resto de las empresas con $11.6 \mathrm{mdp}$ por ineficiencia técnica y 59.3 mdp ineficiencia asignativa. 
Tabla 2

Comparativo respecto a las empresas eficientes del grupo en el 2012, miles de pesos

\begin{tabular}{|c|c|c|c|c|c|c|}
\hline \multicolumn{2}{|l|}{ Empresa } & \multirow{2}{*}{$\begin{array}{c}\begin{array}{c}\text { Cambio } \\
\text { en el costo } \\
\text { de los } \\
\text { insumos }\end{array} \\
-5823375\end{array}$} & \multirow{2}{*}{$\begin{array}{l}\begin{array}{l}\text { Efecto } \\
\text { precio }\end{array} \\
7324186\end{array}$} & \multirow{2}{*}{$\begin{array}{c}\text { Escala } \\
-13218500\end{array}$} & \multirow{2}{*}{$\begin{array}{r}\begin{array}{c}\text { Efecto } \\
\text { eficiencia } \\
\text { técnica }\end{array} \\
11650\end{array}$} & \multirow{2}{*}{$\begin{array}{r}\begin{array}{c}\text { Efecto } \\
\text { eficiencia } \\
\text { asignativa }\end{array} \\
59289\end{array}$} \\
\hline Metlife & Promedio & & & & & \\
\hline & $\begin{array}{l}\text { Des. } \\
\text { estándar }\end{array}$ & 206175 & 6499506 & 6464343 & 34951 & 91073 \\
\hline \multirow[t]{2}{*}{ Quálitas } & Promedio & -8041642 & 4353971 & -12473399 & 36252 & 41535 \\
\hline & $\begin{array}{l}\text { Des. } \\
\text { estándar }\end{array}$ & 811012 & 13913927 & 14007152 & 68937 & 72002 \\
\hline \multirow[t]{2}{*}{$\mathrm{ACE}$} & Promedio & 654608 & -2325405 & 2943940 & 27538 & 8535 \\
\hline & $\begin{array}{l}\text { Des. } \\
\text { estándar }\end{array}$ & 4230328 & 2072486 & 5895734 & 132068 & 40932 \\
\hline \multirow[t]{2}{*}{$\begin{array}{l}\text { B B V A } \\
\text { Bancomer }\end{array}$} & Promedio & -2685360 & 1092109 & -3796957 & 15283 & 4205 \\
\hline & $\begin{array}{l}\text { Des. } \\
\text { estándar }\end{array}$ & 4195262 & 1834066 & 3766899 & 73293 & 20166 \\
\hline \multirow[t]{2}{*}{ GNP } & Promedio & -12259405 & -1278091 & -11005534 & 19046 & 5174 \\
\hline & $\begin{array}{l}\text { Des. } \\
\text { estándar }\end{array}$ & 3371571 & 2651099 & 4254209 & 91341 & 24814 \\
\hline
\end{tabular}

Fuente: elaboración propia.

La tabla 3 nos muestra la descomposición de la brecha generada por el efecto precio dividida en la contribución de los precios por tipo de gasto. En el 2012, Metlife tuvo ventaja en los precios de sus insumos agrupados en los gastos de administración (3.027 mdp) y en los gastos de adquisición (4 415 mdp), no así en los gastos de ajuste, donde los precios que paga Metlife son ligeramente superiores al promedio de su grupo.

En el grupo de empresas de no vida, Quálitas fue la única empresa en cumplir el criterio de ser eficiente en costo de 2008 a 2012. Al igual que Metlife, es la empresa más grande de su grupo, con $43 \%$ de las primas emitidas y de 
los activos fijos. La brecha en costo promedio y la desviación estándar de la tabla 2 muestran que las empresas del grupo tienen menores costos totales (-8 $041 \mathrm{mdp}$ ) con mayor dispersión respecto a las empresas de vida (811 mdp vs 206 mdp). Quálitas, al igual que Metlife opera con mejores precios de sus insumos (4 354 mdp) y las empresas del grupo no vida tienen, en promedio, una brecha en costo por su ineficiencia técnica de 36 mdp y 41 mdp por ineficiencia en la mezcla de sus insumos. A diferencia de Metlife, Quálitas paga menores precios solamente en los insumos agrupados en los gastos de administración, la tabla 3 apunta precios más altos que el promedio de su grupo en los gastos de adquisición (-332 mdp) y en los gastos de ajuste (-25 mdp).

\section{Tabla 3}

Descomposición del efecto precio por tipo de gasto miles de pesos de 2012

\begin{tabular}{|c|c|c|c|c|}
\hline \multicolumn{2}{|l|}{ Empresa } & \multirow{2}{*}{$\begin{array}{c}\begin{array}{c}\text { Efecto precio } \\
\text { gastos de } \\
\text { administración }\end{array} \\
3027913\end{array}$} & \multirow{2}{*}{$\begin{array}{c}\begin{array}{c}\text { Efecto precio } \\
\text { gastos de } \\
\text { adquisición }\end{array} \\
4415927\end{array}$} & \multirow{2}{*}{ 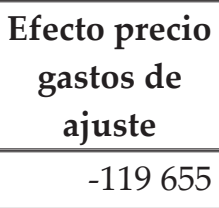 } \\
\hline Metlife & Promedio & & & \\
\hline & Des. Estándar & 4873259 & 4559327 & 23058 \\
\hline \multirow[t]{2}{*}{ Quálitas } & Promedio & 4712460 & -332741 & -25749 \\
\hline & Des. Estándar & 13529864 & 901191 & 1530151 \\
\hline \multirow[t]{2}{*}{$\mathrm{ACE}$} & Promedio & -1780963 & -615864 & 71421 \\
\hline & Des. Estándar & 1681684 & 643735 & 123758 \\
\hline \multirow{2}{*}{$\begin{array}{l}\text { BBVA } \\
\text { Bancomer }\end{array}$} & Promedio & 214511 & 847347 & 30251 \\
\hline & Des. Estándar & 1189762 & 267467 & 267467 \\
\hline \multirow[t]{2}{*}{ GNP } & Promedio & -1040462 & -402385 & 164756 \\
\hline & Des. Estándar & 1358563 & 1888456 & 505368 \\
\hline
\end{tabular}

Fuente: elaboración propia.

En el grupo de las empresas mixtas, varias empresas calificaron para ser la empresa eficiente del grupo: ACE, La Peninsular, Seguros Banamex, Seguros BBVA Bancomer y Tokio Marine. Siendo tres de ellas empresas muy pequeñas y las otras dos bancaseguros, se decidió agregar a GNP a 
las empresas candidatas, por haber sido eficiente en tres de los cinco años y por ser la empresa con mayor venta del grupo. El análisis finalmente se realizó con tres empresas: ACE como representante de las empresas de menor dimensión con $1.7 \%$ de las primas emitidas del grupo; GNP por su ser la empresa más grande del grupo con $19 \%$ de las primas emitidas; y BBVA Bancomer por superar ligeramente en primas a Seguros Banamex con el $9 \%$ de las primas emitidas.

El análisis de la brecha en costos respecto a ACE arroja, como era esperado, su menor escala con el signo positivo de la brecha por 2943 mdp y precios superiores al grupo en 2943 mdp posiblemente asociado a la menor capacidad de negociación y de economías de escala por su tamaño. No hay una clara ventaja en la descomposición del efecto cantidad, salvo las ineficiencias técnica y asignativa promedio de sus pares en $27 \mathrm{mdp}$ y $8 \mathrm{mdp}$. La descomposición del efecto precio de la tabla 3 destaca ventaja en los precios de los gastos de ajuste, por ser el único componente con signo positivo (71 mdp), en tanto el efecto precio de los gastos de administración (-1 780 mdp) y de adquisición (-615 mdp) destacan su desventaja en precios de sus insumos.

El análisis con BBVA Bancomer establece que su costo es mayor al resto de las empresas principalmente por su escala (-3 $796 \mathrm{mdp})$, habla además de menores precios de sus insumos con relación al grupo de las empresas mixtas por la brecha promedio de $1092 \mathrm{mdp}$, confirmada en la tabla 3 con el signo positivo en el efecto precio de los tres tipos de gasto (214 847 y 30 mdp). BBVA Bancomer posiblemente consigue mejores precios por su escala y economías de alcance con el grupo financiero al que pertenece.

El último análisis del grupo de las mixtas, con GNP como empresa eficiente, revela una historia algo diferente. El costo de GNP es mayor al promedio del grupo al ser la empresa con mayor venta del grupo con -12 259 mdp de diferencia. En lo que respecta a los precios, muestra una desventaja en comparación al resto de las compañías Mixtas por -1 278 mdp, en especial en gastos de administración por -1040 mdp y en menor medida en gastos de adquisición con $-402 \mathrm{mdp}$, tabla 3 . En gastos de ajuste se destaca por una ventaja de 164 mdp respecto al promedio del grupo, superior a sus pares ACE y BBVA Bancomer. La ineficiencia promedio del resto de las empresas es muy similar a la que guardan en el comparativo con BBVA Bancomer.

El análisis entre empresas eficientes y sus pares revela ventajas principalmente por el manejo del precio de sus insumos, posiblemente relacionado con economías de escala, además de su ventaja en la eficiencia técnica 
y asignativa. Queda a las aseguradoras el reto de reducción de costos, en especial para las de menor tamaño, a partir de la gestión de las variables endógenas, por ejemplo, haciéndose más eficientes técnicamente al reducir el desperdicio, o buscando la combinación óptima entre sus insumos y precios para llegar a su mínimo costo en la tecnología de producción vigente en la industria.

\section{Análisis de las empresas eficientes del 2008 al 2012}

El análisis transversal descrito en la sección V se extendió aplicándose año tras año del 2008 al 2012 para observar la evolución de las empresas eficientes. El objetivo que se plantea es observar la descomposición ex post de los costos para deducir tendencias en el desempeño de estas compañías. En la figura 2 aparecen las gráficas con la descomposición de los costos durante cinco años para cada empresa eficiente. En la figura 3, se muestra para el mismo periodo, la descomposición del efecto precio por tipo de gasto.

Resalta Metlife en la figura 2.a, por la tendencia en los últimos cinco años a mejorar sus costos por mejores precios con la línea punteada del efecto precio, entre más se aleja del eje horizontal mayor es su ventaja respecto a su grupo. La figura 3.a nos da más información, mostrando que los precios son mejores principalmente en los gastos de administración, una reducción en los costos de adquisición del 2010 al 2012 y casi nula diferencia en los gastos de ajuste. La línea punteada del efecto escala de la figura 2.a al aumentar su valor negativo muestra crecimiento en la operación de la compañía. La información induce a pensar que esta empresa crece con ventajas en precio por su escala.

Quálitas, en un patrón similar a Metlife, denota un ritmo de crecimiento de sus actividades importante con una línea punteada del efecto escala casi recta debajo del eje horizontal en la figura 2.b; salvo en el 2009, mantiene ventaja en precios sobre el promedio de su grupo. En lo que respecta a la descomposición del efecto precio, Quálitas ha mejorado principalmente los precios de los insumos agrupados en sus gastos de administración, con precios ligeramente más altos en gastos de ajuste y de adquisición, como muestra la figura 3.b.

ACE al contrario de Metlife y Quálitas, tiene las curvas del efecto precio por debajo del eje horizontal y la de escala por encima, lo que significa que paga precios más altos y tiene menor tamaño respecto al promedio del grupo. 
Se observa que la empresa crece y a su vez está disminuyendo su brecha respecto a su grupo en precios, si la tendencia continúa, queda abierta la pregunta si esta empresa logrará superar por su tamaño el promedio de su grupo y conseguirá mejores precios que el promedio. De 2009 a 2012 ha ido mejorando la brecha en precios de los insumos de sus gastos de administración como estrategia principal para reducir sus costos como se observa en la figura 3.c., los gastos de ajuste están prácticamente en la media del mercado y los precios de sus gastos de adquisición se han mantenido por arriba del promedio de su grupo.

En menor medida que Metlife o Quálitas, BBVA Bancomer en los últimos cinco años continúa ganando ligera ventaja en precio y tamaño en su grupo. De acuerdo a la figura 3.d, esta empresa ha reducido sus costos a través de los precios de los insumos de sus gastos de administración y ajuste. Sus precios de los gastos de adquisición son mucho mejores que el resto del mercado, lo cual es comprensible si al ser una bancaseguros el contacto con clientes es a través del personal de las sucursales, y no a partir de una fuerza de ventas independiente cuyo ingreso depende totalmente de los bonos y comisiones.

GNP muestra una situación sin grandes cambios, el efecto precio al estar por debajo del eje horizontal significa mayores precios que el promedio del grupo, con mejora en 2009 y 2011 contribuyendo a reducir sus costos en esos mismos años, como señala la forma curveada de la línea del cambio en el costo de sus insumos de la figura 2.e. El efecto escala nos dice que el tamaño de GNP se ha mantenido relativamente estable durante estos cinco años, y no se observa crecimiento en la actividad de la aseguradora en relación a su grupo. La figura 3.e con la descomposición del efecto precio destaca mejores precios en los gastos de ajuste, deterioro en los precios de los gastos de administración en relación al grupo de las empresas mixtas, salvo año 2009, y mayores precios en los gastos de adquisición, sin una tendencia clara de mejora por la caída importante en 2012.

\section{Conclusiones}

En esta investigación se analizaron las diferencias en el costo entre empresas eficientes y sus pares de 2008 a 2012, con un modelo de descomposición apoyado en el análisis de frontera no paramétrico DEA.

El modelo capturó las ventajas de las empresas eficientes, con estimaciones de las brechas en relación a sus pares, en términos monetarios. Metlife y 
Quálitas, empresas seleccionadas para los grupos de vida y no vida respectivamente, tienen desempeño superior a partir de su escala, con menores precios en sus insumos, además de ser eficientes técnica y asignativamente respecto a sus pares. El análisis de 2008 a 2012, muestra, además, una tendencia sostenida que expande la brecha en su grupo por su tamaño y menores precios, en especial en sus gastos de administración.

En cambio, en el grupo de las empresas mixtas resulta interesante que no existira un claro líder. ACE, aun siendo eficiente, paga precios más altos que el promedio del mercado, y en la revisión de cinco años se observa que va consiguiendo mejoras en su desempeño. BBVA Bancomer, por su parte, aventaja al grupo por su escala y precios en los tres tipos de gastos, no es sorprendente por ser una bancaseguros con mayor posibilidad de aprovechar economías de alcance. Por último GNP, aunque es la empresa de mayor tamaño del grupo, no presenta como Metlife y Quálitas beneficios en costos por los precios de sus insumos, por el contrario, su precios son superiores en promedio a sus pares, y el análisis de cinco años recalca que la brecha en precios se amplía. Varias explicaciones pueden justificar esta brecha, por ejemplo, en promedio, el grupo puede estar mejorando sus precios, la empresa no ha conseguido aprovechar su escala para mejorar los precios de sus insumos o maneja estándares superiores a la media del mercado.

En todos los grupos hay empresas ineficientes en costo y, de acuerdo a los resultados, la ineficiencia promedio se ha mantenido en los últimos cinco años. Queda el reto a los accionistas para crear los incentivos que induzcan a los administradores a buscar márgenes superiores a través de mejorar la eficiencia en costo; a los administradores para gestionar las variables a su alcance para reducir el desperdicio y ser más competitivos en precio; y a reguladores para crear las condiciones que induzcan a las instituciones de seguros a reducir su ineficiencia y mejorar su productividad y de esta forma transferir a la sociedad el beneficio en precio que da la optimización en los costos a través del cambio tecnológico y la eficiencia en costo. 


\section{Referencias}

Accenture (2014). Big opportunities in small-ticket insurance. Referencia 14-1126 / 11-7492. Banco de México (2012- 2013). Reporte sobre el Sistema Financiero, México.

Barros, C.; N. Barroso y M. Borges (2005). “Evaluating the Efficieny and Productivity of Insurance Companies wirh Malmquist Index: A Case of Study for Portugal", The Geneva Papers, pp. 244-267.

Barros, C.; A. Iblwoye y S. Managl (2008). "Productivity Change of Nigerian Insurance Companies: 1994 -2005". African Development Review, pp. 505-528.

Ben-Shahar, O.; y K. D. Logue (2012). Outsourcing regulation: How Insurance reduces moral hazard. Michigan Law Review, vol. 111, No. 2, pp. 197-248.

Bernstein, J. (1999). "Total factor productivity growth in the Canadian life insurance industry 1979-1989", Canadian Journal of Economics, vol. 32, No. 2, pp. 500-517.

Borenstein, S. y J. Farrell (2000). "Is Cost-cutting Evidence of X-inefficiency?", American Economic Review, vol. 90, pp. 224-227.

Comisión Nacional de Seguros y Fianzas (2002). “Ley General de Instituciones y Sociedades Mutualistas de Seguros". Diario Oficial de la Federación, México.

Comisión Nacional de Seguros y Fianzas (2000-2011a). Anuario Estadístico de Seguros y Fianzas, México.

Comisión Nacional de Seguros y Fianzas (2000-2013b). Boletín de Análisis Sectorial, México.

Comisión Nacional de Seguros y Fianzas (2011c). Circular Modificatoria 6/11 de la Única de Seguros, México.

Cummins, D. y O. Mahul (2009). Catastrophe Risk Financing in Developing Countries. Washington, D.C: The World Bank.

Cummins, D. y M. Weiss (1993). “Measuring cost efficiency in the property-liability insurance industry", Journal of Banking and Finance, vol. 17, pp. 463-481.

(1998). Analyzing Firm Performance in the Insurance Industry Using Frontier Efficiency Methods, Working paper, The Wharton School, University of Pennsylvania.

Diewert, W. (2005). "Index number theory using differences rather than ratios", American Journal of Economics and Sociology, vol. 64, pp. 311-360.

Fuentes, H. (1993). Análisis y perspectivas del sector asegurador mexicano. Universidad Panamericana, Escuela de Economía, México: Universidad Panamericana, Escuela de Economía.

Fuentes, H., Grifell-Tatjè E. y Perelman S. (2001). "A Parametric Distance Function Approach for Malmquist Productivity Index Estimation", Journal of Productiviy Analysis, vol. 15, pp. 79-94. 
(2005). Product Specialization, Efficiency and Productivity Change in the Spanish Insurance Industry, Working paper, Université de Liège, Bélgica.

Fukuyama, H. y W. Weber (2001). "Efficiency and Productivity Change of Non-Life Insurance Companies in Japan”. Pacific Economic Review, vol. 6, No. 1, 129-146.

Garg, M. y Deepti (2008). "Efficiency of General Insurance Industry in India in the Post-Liberalization Era: A Data Envelopment Approach". Journal of Risk \& Insurance, vol. 1, pp. 32-49.

Grifell-Tatjé E. y K. Lovell (1999). "Profits and Productivity", Management Science, vol. 45, No. 9, pp.1177-1193.

E. y C.A. Lovell (2000). "Costs and Productivity". Managerial and Decision Economics, vol. 21, pp. 19-30.

Hardwick, P. y W. Dúo (1998). “The Competitiveness of EU Insurance Industries”. The Service Industries Journal, vol. 18, No. 1, pp. 39-54.

Huidobro, A. (2002). Economías de Escala y de Alcance en el Sector Asegurador Mexicano. México, tesis maestría en economía ITESM CCM.

Jameti, M. y T. Von Urgern-Sternberg (2005). "Assesing the Efficiency of an Insurance Provider-A Measurement Error Approach". The Geneva Risk and Insurance Review. vol. 30, pp.15-34.

Kader, H., M. Adams y P. Hardwick (2010). "The Cost Efficiency of Takaful Insurance Companies". The Geneva Papers, vol 35, pp. 161-181.

Kasman, A. y E. Turgutlu (2009). "Total Factor Productivity in the Turkish Insurance Industry", International Journal of the Economics of Business, pp. 239-247.

Kumbhakar, S. y K. Lovell (2003). Stochastic Frontier Analysis. Reino Unido: Cambridge University Press.

Lim, S. y K. Lovell (2008). "Short-Run Total Cost Change and Productivity of US Class I Railroads". Journal of Transport Economics and Policy. vol. 41, No. 1, pp. 155-188.

Lovell, K. (2008). "Production frontiers and productivity efficiency". En Fried et al. (2008) The Measurement of Productivity Efficiency, Techniques and Applications. EUA: Oxford University Press, pp. 3-67.

Mahlberg, B. y T. Url (2003). "Effects of the single market on the Austrian insurance industry". Empirical Economics. vol. 28, pp. 213-838.

MarketLine (2014). Life Insurance in Mexico. Referencia 0071-0976. Reino Unido.

Rai, Anoop (1996). "Cost Efficiency of International Insurance Firms", Journal of Financial Services Research, vol. 10, pp. 213-233. 
Sahoo, B. y K. Tone (2009). “Radial and non-radial decompositions of profit change: With an application to Indian banking", European Journal of Operational Research., vol. 196, pp. 1130-1146.

Seiford, L.M. y J. Zhu (2002). "Modeling undesirable factors in efficiency evaluation", European Journal of Operational Reseach. vol. 142, pp.16-20.

Schlesinger, H. y E. Venezian (1986). "Insurance markets with loss-prevention activity: profits, market structure, and consumer welfare", Rand Journal of Economics, vol. 17, No. 2, pp. 227-238.

Trigo-Gamarra, L. y C. Growitsch (2010). "Comparing Single and Multichannel Distribution Strategies in the German Live Insurance Market: an analysis of cost and profit efficiency", Schmalenbach Business Revise, vol. 62, pp. 401-417.

Triplett, J. y B. Boswordth (2004). Productivity in the U.S. Services Sector: new sources of economic growth, Brookings Institution Press.

Turchetti, G. y C. Daraio (2004). "How Deregulation Shapes Market Structure and Industry Efficiency: The Case of the Italian Motor Insurance Industry", The Geneva Papers on Risk and Insurance. vol. 29, No. 2, pp. 202-218

Ugalde, U. (1993). La función de costos en el sector asegurador mexicano: una primera aproximación a la evidencia sobre economías de escala y economías de alcance, tesis de Licenciatura en Economía, ITAM.

Varian, H. (1992). Microeconomic Analysis. Nueva York: W.W. Norton \& Company.

Weiss, M. (1986). "Analysis of Productivity at the Firm Level: An Application to Life insurers", Journal of Risk Insurance, vol. 53, No. 1, pp. 49-83.

Wolff (1991). "Productivity Growth, Capital Intensity, and Skill Levels, in the U.S. Insurance Industry, 1948-86", The Geneva Papers on Risk and Insurance, pp. 173-190.

Zhu, J. (2009). Quantitative Models for Performance Evaluation and Benchmarking. EUA: Springer. 
Anexo

Figura 2

Evolución de la brecha de las empresas eficientes

2a) Comparativo respecto a Metllife

2a) Comparativo respecto a Qualitas

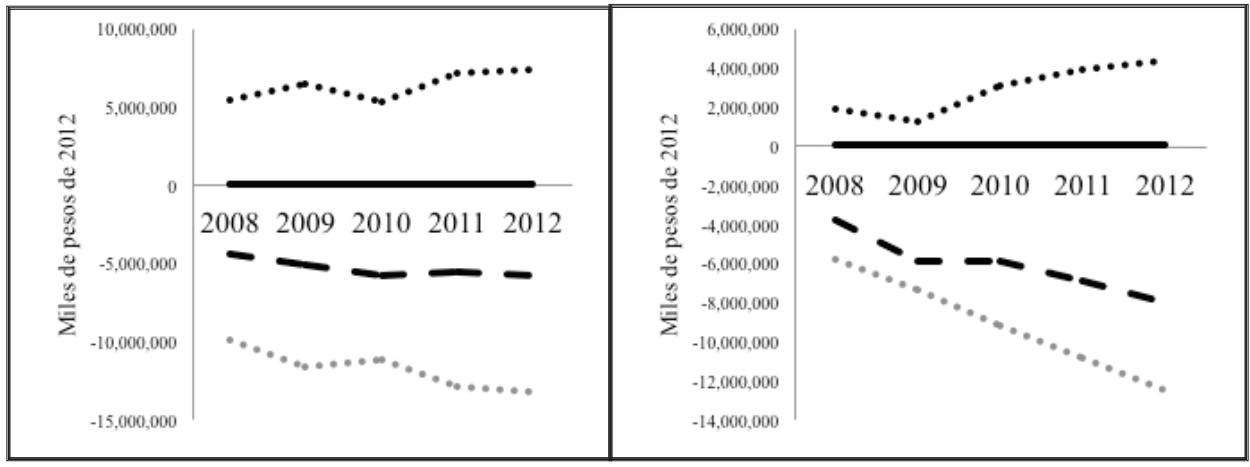

2c) Comparativo respecto a $\mathrm{ACE}$

2d Comparativo respecto a BBVA Bancomer

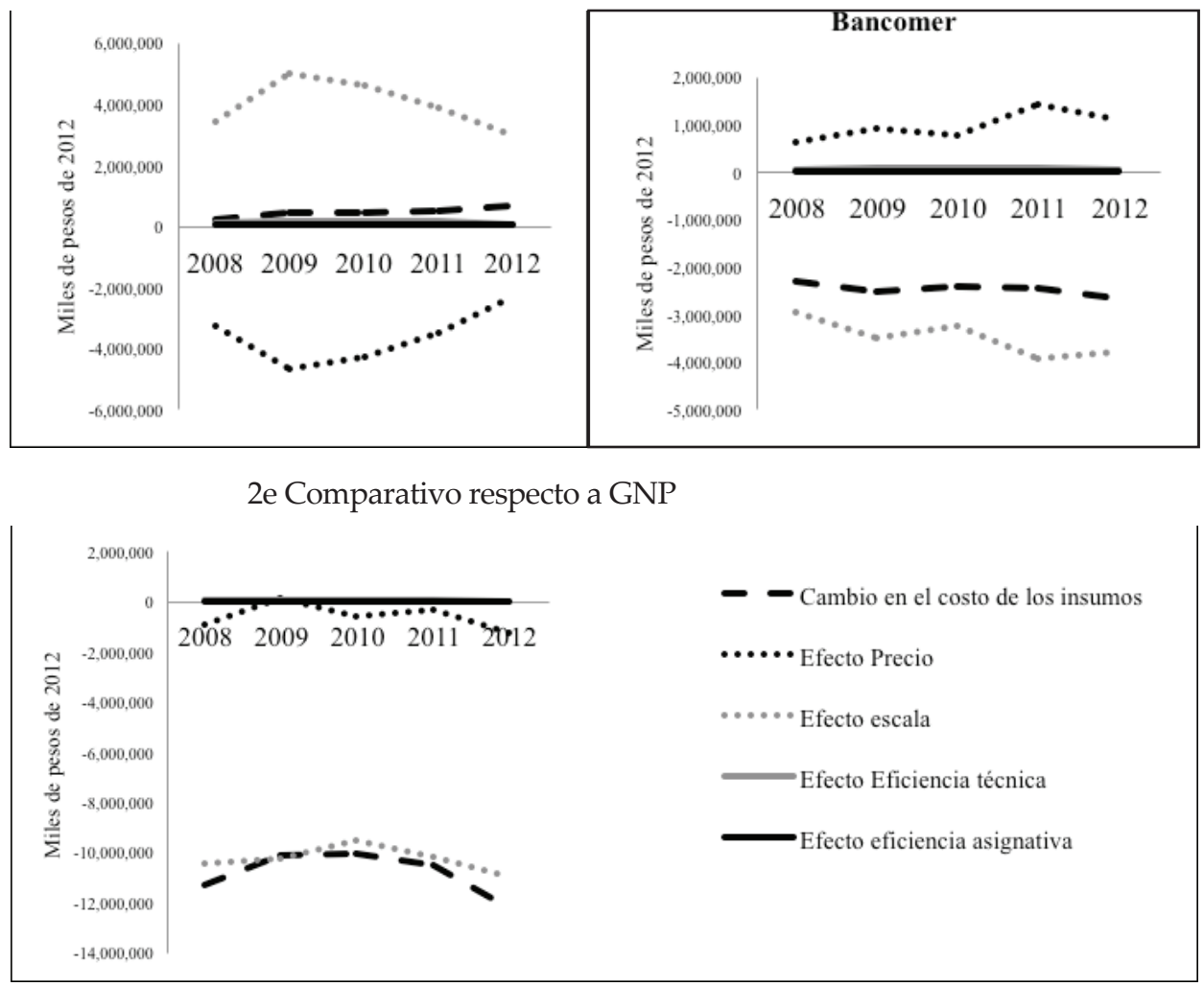




\section{Figura 3}

Evolución del efecto precio de los gastos de las empresas eficientes

3a) Descomposición efecto precio Metlife

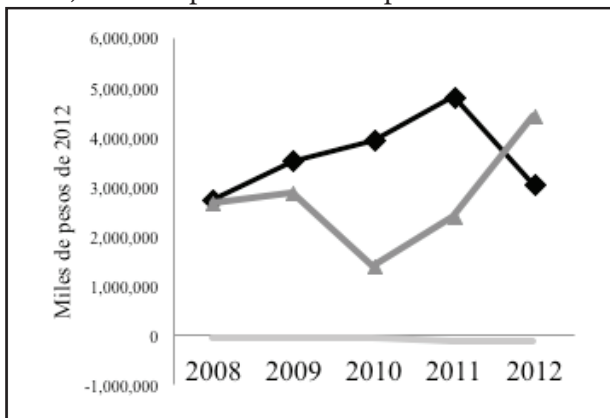

3c) Descomposición efecto precio ACE

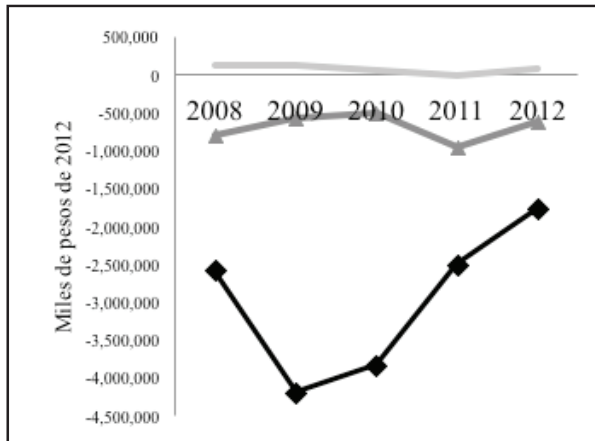

3b) Descomposición efecto precio Quálitas

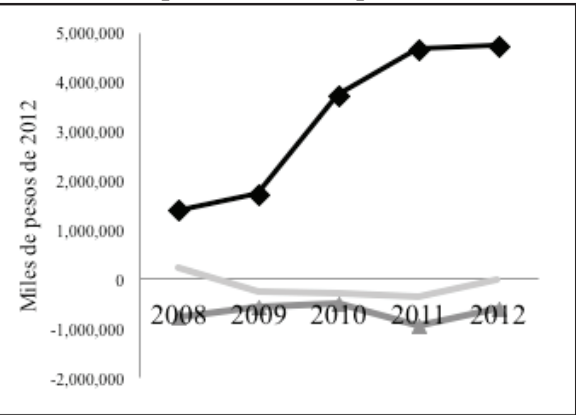

3d) Descomposición efecto precio BBVA Bancomer

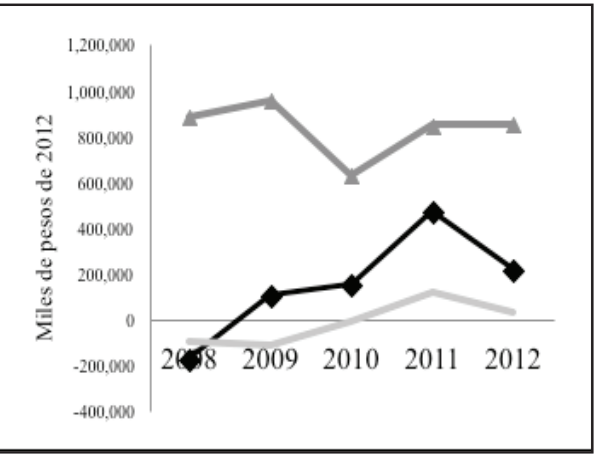

3b) Descomposición efecto precio GNP

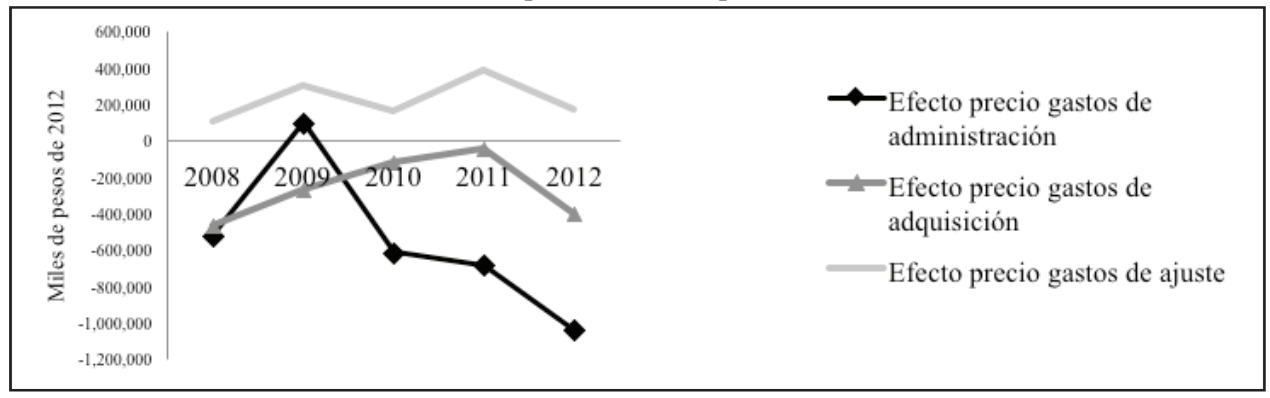

